\title{
uvby COLOURS OF CEPHEID VARIABLES
}

\author{
R. A. BELL
}

Dept. of Physics and Astronomy, University of Maryland, College Park, Md., U.S.A.

\begin{abstract}
Synthetic stellar spectra have been computed for Cepheids and supergiant $\mathrm{F}$ and $\mathrm{G}$ stars, using flux constant model atmospheres. These spectra have been used to compute theoretical $u v b y$ and $U B V$ colours which may be compared with the observed colours. The supergiant $\alpha$ Per is used to establish the zero points of the theoretical colours. The colour comparison can be used to determine the temperatures, gravities and metal abundances of the stars as well as an estimate of the interstellar reddening. The reddening determinations are carried out by arguing that the temperature and gravity values obtained from $U-B$ and $B-V$ must match those obtained from $c_{1}$ and $b-y$.

A more detailed account of this work will be given elsewhere.
\end{abstract}

\section{DISCUSSION}

Tayler: Are not those gravity variations that you've got there really unbelievably large? A factor of 4 in gravity means a factor of 2 in radius.

Bell: Well, there's acceleration terms; it is an effective gravity, not a static one. The radius change would be so small that you would get practically no change in gravity from that effect alone.

Rodgers: Yes, you are in fact measuring a pressure here, and this is the pressure through the photosphere. That's what the effective gravity is about; that's where the factor of 2 is understandable. 